International Journal of Pure and Applied Mathematics

Volume 110 No. 3 2016, 489-501

ISSN: 1311-8080 (printed version); ISSN: 1314-3395 (on-line version)

url: http://www.ijpam.eu

doi: 10.12732/ijpam.v110i3.9

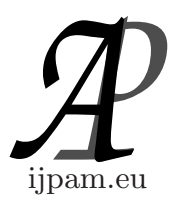

\title{
K-QUASI CLASS Q AND k-QUASI * CLASS \\ Q COMPOSITION OPERATORS ON \\ WEIGHTED HARDY SPACE
}

\author{
A. Devika ${ }^{1}$, G. Suresh ${ }^{2}$ \\ ${ }^{1,2}$ PSG College of Arts and Science \\ Coimbatore, 14, INDIA
}

\begin{abstract}
In this paper we discuss the conditions for a composition operator and a weighted composition operator to be $\mathrm{k}$ quasi class $\mathrm{Q}$ and $\mathrm{k}$ quasi * class $\mathrm{Q}$ operator and also the characterization of $\mathrm{k}$ quasi class $\mathrm{Q}$ and $\mathrm{k}$ quasi * class $\mathrm{Q}$ composition operators on weighted Hardy space.
\end{abstract}

AMS Subject Classification: 47B20, 47B99, 47B15

Key Words: Hilbert space, quasi * class Q operators, composition operators, Hardy space

\section{Introduction}

Various properties of composition operators on weighted Hardy spaces have been studied by different authors [3] Cowen and Kriete obtained a nice correlation between hyponormality of composition operator on $H^{2}$. [9] E.A. Nordgeen, studied some results on the hyponormality of composition operators and their adjoints. [13] S. Panayappan D.Senthil kumar and Mohenraj have investigated on M-Quasihyponormality of composition operators and their adjoints. [15] T. Veluchamy have investigated parahyponormal * paranormal and posinormal operators.

Received: $\quad$ August 11, 2016

Revised: September 26, 2016

Published: November 5, 2016

$\S_{\text {Correspondence author }}$ (c) 2016 Academic Publications, Ltd. url: www.acadpubl.eu 
In this paper, we are interested in k-quasi * class Q Operators, we give a characterization of such operators and other known classes of operators

\section{Preliminary Notes}

Let $\mathrm{f}$ be an analytic map on the open disk $\mathrm{D}$ given by the Taylors series

$$
f(z)=a_{0}+a_{1} z+a_{2} z^{2}+\ldots
$$

Let $\beta=\left\{\beta_{n}\right\}_{n=0}^{\infty}$ be a sequence of positive numbers with $\beta_{0}=1$ and $\frac{\beta_{n+1}}{\beta_{n}} \rightarrow 1$ as $n \rightarrow \infty$. The set $H^{2}(\beta)$ of formal complex power series $f(z)=\sum_{n=0}^{\infty} a_{n} z^{n}$ such that $\|f\|_{\beta}^{2}=\sum_{n=0}^{\infty}\left|a_{n}\right|^{2} \beta_{n}^{2}<\infty$ is a Hilbert space of functions analytic in the unit disk with the inner product $\langle f, g\rangle_{\beta}=\sum_{n=0}^{\infty} a_{n} \overline{b_{n}} \beta_{n}^{2}$ for $\mathrm{f}$ as above and $g(z)=\sum_{n=0}^{\infty} b_{n} z^{n}$.

Let $\mathrm{D}$ be the open unit disk in the complex plane and let $T: D \rightarrow D$ be an analytic self map of the unit disk and consider the corresponding composition operator $C$ acting on $H^{2}(\beta)$ ie., $C f=f o T, f \in H^{2}(\beta)$.

Let $\left(x, \sum, \lambda\right)$ be a sigma-finite measure space and let $T: X \rightarrow X$ be a non singular measurable transformation. A bounded linear operator $C f=f o T$ on $L^{2}\left(x, \sum, \lambda\right)$ is said to be a composition operator induced by $\mathrm{T}$ when the measure $\lambda T^{-1}$ is absolutely continuous with respect to the measure $\lambda$ and the Random-Nikodym derivative $\frac{d \lambda T^{-1}}{d \lambda}=f_{0}$ is essentially bounded. The randomNikodym derivative of the measure $\lambda\left(T^{k}\right)^{-1}$ with respect to $\lambda$ is denoted by $f_{0}^{(k)}$ where $T^{k}$ is obtained by composing T-k times. Every essentially bounded complex valued measurable function $f_{0}$ induces the bounded operator $M_{f_{0}}$ on $L^{2}(\lambda)$ which is defined by $M_{f_{0}} f=f_{0} f$ for every $f \in L^{2}(\lambda)$. Further $C^{*} C=M_{f_{0}}$ and $C^{* 2} C^{2}=M_{f_{0}^{2}}$.

Let $\mathrm{u}$ be an essentially bounded function. Then the weighted composition operator $W\left(=W_{u, T}\right)$ on the space $L^{2}(\mu)$ induced by $\mathrm{u}$ and $\mathrm{T}$ is given by $W f=$ $u$. foT for each $f \in L^{2}(\mu)$.

A transformation $\mathrm{T}$ is measurable if $T^{-1}(A) \in \mathbf{A}$ for any $A \in \mathbf{A}$. A measurable transformation $\mathrm{T}$ is said to be non-singular if $\mu\left(T^{-1}(A)\right)=0$ whenever $\mu(A)=0$ for $A \in \mathbf{A}$. If $\mathrm{T}$ is a measurable transformation then $T^{n}$ is also a measurable transformation. If $\mathrm{T}$ is non-singular, then we say that $\mu T^{-1}$ is absolutely continuous with respect to $\mu$ and hence $\mu\left(T^{-1}\right)^{n}$ becomes absolutely continuous with respect to $\mu$. Hence, by Radon-Nikodym theorem there exists a unique non-negative essentially bounded measurable function $h_{n}$ such that

$$
\mu\left(T^{-1}\right)^{n}(A)=\int_{A} h_{n} d \mu \text { for every } A \in \mathbf{A}
$$


and $h_{n}$ is called the nth order radon-nikodym derivative and is denoted by $\frac{d \mu\left(T^{-1}\right)^{n}}{d \mu}$. It can be seen that $h_{n}=h . h o T^{-1} \cdot h o T^{-2} \ldots h o T^{-(n-1)}$ and $h_{n}=$ $h_{n-1} \cdot h o T^{-(n-1)}$. Throughout this paper, we assume that $\mathrm{u}$ is non-negative.

Definition 2.1. [10]

An operator $T$ on a Hilbert space $H$ is said to be $k$-quasi class $Q$ if

$$
T^{*(k+2)} T^{k+2}-2 T^{*(k+1)} T^{k+1}+T^{* k} T^{k} \geq 0 .
$$

Definition 2.2. An operator $T$ on a Hilbert space $H$ is said to be *-class $Q$ if

$$
T^{* 2} T^{2}-2 T T^{*}+I \geq 0
$$

Definition 2.3. An operator $T$ on a Hilbert space $H$ is said to be quasi*-class $Q$ if

$$
T^{* 3} T^{3}-2\left(T^{*} T\right)^{2}+T^{*} T \geq 0 .
$$

Definition 2.4. An operator $T$ on a Hilbert space $H$ is said to be $k$-quasi*-class $Q$ if

$$
T^{*(k+2)} T^{k+2}-2 T^{* k} T T^{*} T^{k}+T^{* k} T^{k} \geq 0 .
$$

Proposition 1. Change of variable: Let $X$ be a non-empty set and let $\boldsymbol{A}$ be a $\sigma$-algebra on $X$. Let $\mu$ and $\mu T^{-1}$ be measures on $\boldsymbol{A}$ let $h: X \rightarrow[0, \infty]$ be a measurable function. Then the following are equivalent:

(i) $\mu T^{-1}$ is absolutely continuous with respect to $\mu$ and $h$ is Radon-nikodym derivative of $\mu T^{-1}$ with respect to $\mu$.

(ii) For every measurable function $f: X \rightarrow[0, \infty]$, the equality

$$
\int_{X} f d \mu T^{-1}=\int_{X} f h d \mu
$$

holds.

The conditional expectation operator $E\left(T^{-1}(\boldsymbol{A})\right)=E(f)$ is defined for each non-negative function $f$ in $L^{p}(1 \leq p<\infty)$ and is uniquely determined by the following set of conditions:

(i) $E(f)$ is $T^{-1}(\boldsymbol{A})$ measurable. 
(ii) If $B$ is any $T^{-1}(\boldsymbol{A})$ measurable set for which $\int_{A} f d \mu$ converges then we have

$$
\int_{A} f d \mu=\int_{A} E(f) d \mu
$$

$E$ is the projection operator onto the closure of the range of the composition operator $C$ on $L^{2}(\mu)$.

Lemma 1. [14] Let $P$ be the projection of $L^{2}(X, \boldsymbol{A}, \mu)$ onto $\overline{R(C)}$. Then:

(i) $C^{*} C f=h f$ and $C C^{*} f=(h o T) P f$ for all $f \in L^{2}(\mu)$.

(ii) $\overline{R(C)}=\left\{f \in L^{2}(\mu): f\right.$ is $T^{-1}(\boldsymbol{A})$ measurable $\}$.

(iii) If $f$ is $T^{-1}(\boldsymbol{A})$ measurable and $g$ and fg belong to $L^{2}(\mu)$, then $P(f g)=$ $f P(g)$, (f need not be in $L^{2}(\mu)$ ).

In [12] Senthikumar has proved the conditions for composition and weighted composition operators to be k-quasi paranormal operator. A. Gupta and N. Bhatia [1] also proved the conditions for composition and weighted composition operators to be $(\mathrm{n}, \mathrm{k})$-quasi paranormal and $(\mathrm{n}, \mathrm{k})$-quasi -*-paranormal operators. In this paper we obtain the conditions for composition and weighted composition operators to be $\mathrm{k}$ quasi * class $\mathrm{Q}$ operator and quasi * class $\mathrm{Q}$ operators in terms of expectation operator and Radon-Nikodyn derivative $\mathrm{h}$ (or $\left.h_{n}\right)$

\section{Composition Operator}

Let $\mathrm{C}$ be the composition operator and $C^{*}$ be its adjoint which is given by

$$
C^{*} f=h . E(f) o T^{-1} .
$$

Proposition 2. [1] For every $n \in N$ :

(i) $\left(C^{*} C\right)^{n} f=h^{n} f$.

(ii) $\left(C C^{*}\right)^{n} f=(h o T)^{n} P(f)$.

(iii) $E$ is the identity operator on $L^{2}(\mu)$ iff $T^{-1}(\boldsymbol{A})=\boldsymbol{A}$.

Theorem 3.1. Let $C$ be a composition operator on $L^{2}(\mu)$. Then $C$ is $k$-quasi class $Q$ if and only if $h^{k+2}-2 h^{k+1}+h^{k} \geq 0$. 
Proof. Suppose C is k-quasi class Q operator. Then for every $f \in L^{2}(\mu)$,

$$
\left\langle\left(C^{*(k+2)} C^{k+2}-2 C^{*(k+1)} C^{k+1}+C^{* k} C^{k}\right) f, f\right\rangle \geq 0 .
$$

Let $f=\chi_{A}$ with $\mu(A)<\infty$. Therefore

$$
\begin{aligned}
& \left\langle\left(C^{*(k+2)} C^{k+2}-2 C^{*(k+1)} C^{k+1}+C^{* k} C^{k}\right) \chi_{A}, \chi_{A}\right\rangle \geq 0 \\
& \Leftrightarrow \int\left(\left(h^{k+2}-2 h^{k+1}+h^{k}\right) \chi_{A}\right) d \mu \geq 0 \\
& \Leftrightarrow \int\left(h^{k+2}-2 h^{k+1}+h^{k}\right) d \mu \geq 0 \\
& \Leftrightarrow h^{k+2}-2 h^{k+1}+h^{k} \geq 0 .
\end{aligned}
$$

Theorem 3.2. Let $C$ be a composition operator on $L^{2}(\mu)$. Then $C$ is $k$-quasi * class $Q$ if and only if $h^{k+2}-2 h_{k} . E(h) o T^{-1}+h^{k} \geq 0$.

Proof. Consider

$$
\begin{aligned}
C^{* k} C C^{*} C^{k} f & =C^{* k} C C^{*}\left(f o T^{k}\right) \\
& =C^{* k} C\left(h \cdot E\left(f o T^{k}\right) o T^{-1}\right) \\
& =C^{* k}\left(h o T^{-1} \cdot f o T^{k}\right) o T \\
& =h_{k} \cdot E(h) o T^{-1} \cdot f .
\end{aligned}
$$

$\mathrm{C}$ is k-quasi * class Q if and only if for every $f \in L^{2}(\mu)$ and $\lambda>0$,

$$
\begin{aligned}
& \left\langle\left(C^{*(k+2)} C^{k+2}-2 C^{* k} C C^{*} C^{k}+C^{* k} C^{k}\right) f, f\right\rangle \geq 0 . \\
& \Leftrightarrow\left(h^{k+2}-2 h_{k} \cdot E(h) o T^{-1}+h^{k}\right) f \geq 0 \\
& \Leftrightarrow h^{k+2}-2 h_{k} \cdot E(h) o T^{-1}+h^{k} \geq 0 .
\end{aligned}
$$

Example 3.1. Consider the space $l^{2}(\omega)=L^{2}\left(N, 2^{N}, \mu\right)(\omega)$ where $\omega=$ $\left\langle m_{n}\right\rangle_{n=1}^{\infty}$ is a sequence of positive real numbers. $\mu$ is a measure given by $\mu(n)=$ $m_{n}$. Let $T: N \rightarrow N$ be a non-singular measurable transformation. Then $T^{n}$ is also a non-singular measurable transformation for $n \in N$. Now,

$$
\begin{aligned}
h_{k}(s) & =\frac{1}{m_{s}} \sum_{j \in T^{-k}(s)} m_{j} \\
h^{k}(s) & =\frac{1}{m_{s}^{k}}\left(\sum_{j \in T^{-1}(s)} m_{j}\right)
\end{aligned}
$$




$$
E(f)(k)=\frac{\sum_{j \in T^{-1} T(k)} f_{j} m_{j}}{\sum_{j \in T^{-1} T(k)} m_{j}}
$$

for all non-negative sequence $f=<f_{n}>_{n=1}^{\infty}$ and $s, k \in N$. by theorem (3.1), C is k-quasi class $\mathrm{Q}$ if and only if

$$
\begin{array}{r}
\frac{1}{m_{s}^{k+2}}\left(\sum_{j \in T^{-1}(s)} m_{j}\right)^{k+2}-2 \frac{1}{m_{s}^{k+1}}\left(\sum_{j \in T^{-1}(s)} m_{j}\right)^{k+1} \\
+\frac{1}{m_{s}^{k}}\left(\sum_{j \in T^{-1}(s)} m_{j} \geq 0 .\right.
\end{array}
$$

for all non-negative sequence $f=<f_{n}>_{n=1}^{\infty}$ and $s, k \in N$. by theorem (3.2), C is $\mathrm{k}$-quasi * class $\mathrm{Q}$ if and only if

$$
\begin{array}{r}
\frac{1}{m_{s}^{k+2}}\left(\sum_{j \in T^{-1}(s)} m_{j}\right)^{k+2}-2 \frac{1}{m_{s}} \sum_{j \in T^{-k}(s)} m_{j} \frac{1}{m_{T^{-k}(s)}} \sum_{j \in T^{-k+1}(s)} m_{j} \\
+\frac{1}{m_{s}^{k}}\left(\sum j \in T^{-1}(s) m_{j}\right)^{k} \geq 0 .
\end{array}
$$

Proposition 3. If $P$ denote the projection of $L^{2}(\mu)$ on $\overline{R(C)}$, then $C^{*} C f=f_{0} f$ and $C C^{*} f=\left(f_{0} o T\right) P f$. For all $f \in L^{2}(\mu)$, where $P$ denote the projection of $L^{2}$ on $\overline{R(C)}$ and

$$
\overline{R(C)}=\left\{f \in L^{2}: f \text { is } T^{-1} \sum \text { measurable }\right\} .
$$

Theorem 3.3. Let $C \in B\left(L^{2}(\mu)\right)$. Then $C$ is of quasi * class $Q$ if and only if

$$
f_{o}^{(3)}-2\left(f_{0}\right)^{2}+f_{0} \geq 0 .
$$

Proof. Let $C \in B\left(L^{2}(\mu)\right)$. Then $\mathrm{C}$ is of quasi * class $\mathrm{Q}$ operator

$$
\begin{aligned}
& \Leftrightarrow C^{* 3} C^{3}-2\left(C^{*} C\right)^{2}+C^{*} C \geq 0 \\
& \Leftrightarrow\left\langle\left(C^{* 3} C^{3}-2\left(C^{*} C\right)^{2}+C^{*} C\right) \chi_{E}, \chi_{E}\right\rangle \geq 0
\end{aligned}
$$

for every characteristic function $\chi_{E}$ of $E$ in $\sum$ such that $\lambda(E) \prec \infty$.

Since, $C^{* 2} C^{2}=M_{f_{0}^{(2)}}, C^{*} C=M f_{0}$.

$$
\Leftrightarrow\left\langle\left(M_{f_{0}^{(3)}}-2\left(M_{f_{0}}\right)^{2}+M_{f_{0}}\right) \chi_{E}, \chi_{E}\right\rangle \geq 0
$$




$$
\Leftrightarrow \int_{E}\left(f_{0}^{(3)}-2\left(f_{0}\right)^{2}+f_{0}\right) d \mu \geq 0
$$

for every $\mathrm{E}$ in $\sum$.

Hence $\mathrm{C}$ is quasi ${ }^{*}$ class $\mathrm{Q}$ operator if and only if $f_{o}^{(3)}-2\left(f_{0}\right)^{2}+f_{0} \geq 0$.

Corollary 1. Let $C \in B\left(L^{2}(\mu)\right)$ with dense range. Then $C \in$ quasi * class $Q$ if and only if $f_{o}^{(3)}-2\left(f_{0}\right)^{2}+f_{0} \geq 0$

Theorem 3.4. Let $C \in B\left(L^{2}(\mu)\right)$. Then $C^{*} \in$ quasi * class $Q$ if and only if

$$
\left(f_{0} o T\right)^{3} P_{1}-2\left(\left(f_{0} o T\right) P_{1}\right)^{2}+\left(f_{0} o T\right) P_{1} \geq 0
$$

where $P_{1}$ is the projection of $L^{2}$ on to $\overline{R(C)}$.

Proof. Let $C^{*}$ is of quasi * class Q operator if and only if

$$
\begin{aligned}
& C^{3} C^{* 3}-2\left(C C^{*}\right)^{2}+C C^{*} \geq 0, \\
& \left\langle\left(C^{3} C^{* 3}-2\left(C C^{*}\right)^{2}+C C^{*}\right) f, f\right\rangle \geq 0
\end{aligned}
$$

for every $f \in L^{2}$. $\left\langle C C^{*} f, f>=<\left(f_{0} o T\right) P_{1} f, f>\right.$ where $P_{1}$ is the projections of $L^{2}$ on to $\overline{R(C)}$. Thus $C^{*}$ is of quasi * class Q Operator if and only if

$$
\left\langle\left(f_{0} o T\right)^{3} P_{1} f, f\right\rangle-2\left\langle\left(\left(f_{0} o T\right) P_{1}\right)^{2} f, f\right\rangle+\left\langle\left(f_{0} o T\right) P_{1} f, f\right\rangle \geq 0
$$

for every $f \in L^{2}$,

$$
\left(f_{0} o T\right)^{3} P_{1}-2\left(\left(f_{0} o T\right) P_{1}\right)^{2}+\left(f_{0} o T\right) P_{1} \geq 0 .
$$

Corollary 2. Let $C \in B\left(L^{2}(\mu)\right)$ with dense range. Then $C \in$ quasi * class $Q$ operator if and only if $\left(f_{0} o T\right)^{3} P_{1}-2\left(\left(f_{0} o T\right) P_{1}\right)^{2}+\left(f_{0} o T\right) P_{1} \geq 0$

\section{Weighted Composition Operators}

Let $\mathrm{W}$ be the weighted composition operator on $L^{2}(\mu)$. Let $W^{*}$ be its adjoint which is given by $W^{*} f=h . E(u . f) o T^{-1}$ for $f \in L^{2}(\mu)$. For a positive integer $\mathrm{n}, u_{n}=u \cdot(u o T)^{2} \ldots(u o T)^{(n-1)}$. For $f \in L^{2}(\mu), W^{n} f=u_{n} . f o T^{-n}$ and $W^{* n} f=$ $h_{n} \cdot E\left(u_{n} \cdot f\right) o T^{-n}$. 
Proposition 4. [2] For $u \geq 0$ :

(i) $W^{*} W f=h E\left[\left(u^{2}\right)\right] o T^{-1} f$.

(ii) $W W^{*} f=u(h o T) E(u f)$.

Theorem 4.1. Let $W$ be a weighted composition operator on $L^{2}(\mu)$. Then $W$ is k-quasi class $Q$ operator if and only if

$$
h_{k+2} \cdot E\left(u_{k+2}^{2}\right) o T^{-(2)}-2 h_{k+1} \cdot E\left(u_{k+1}^{2}\right) o T^{-1}+h_{k} \cdot E\left(u_{k}^{2}\right) \geq 0 .
$$

Proof. Suppose W is k-quasi class Q operator. Then for $f \in L^{2}(\mu)$,

$$
\left\langle\left(W^{* k} W^{* 2} W^{2} W^{k}-2 W^{* k} W^{*} W W^{k}+W^{* k} W^{k}\right) f, f\right\rangle \geq 0 .
$$

Let $f=\chi_{A}$ with $\mu(A)<\infty$. Then

$$
\begin{aligned}
& \left\langle\left(W^{* k} W^{* 2} W^{2} W^{k}-2 W^{* k} W^{*} W W^{k}+W^{* k} W^{k}\right) \chi_{A}, \chi_{A}\right\rangle \geq 0, \\
& \Leftrightarrow\left\langle\left( h_{k+2} \cdot E\left(u_{k+2}^{2}\right) o T^{-(k+2)}-2 h_{k+1} \cdot E\left(u_{k+1}^{2}\right) o T^{-(k+1)}\right.\right. \\
& \left.\left.\quad+h_{k} \cdot E\left(u_{k}^{2}\right) o T^{-k}\right) \chi_{A}, \chi_{A}\right\rangle \geq 0 \\
& \Leftrightarrow \int\left(h_{k+2} \cdot E\left(u_{k+2}^{2}\right) o T^{-(k+2)}-2 h_{k+1} \cdot E\left(u_{k+1}^{2}\right) o T^{-(k+1)}\right. \\
& \left.\quad+h_{k} \cdot E\left(u_{k}^{2}\right) o T^{-k}\right) \chi_{A} d \mu \geq 0 \\
& \Leftrightarrow \int h_{k+2} \cdot E\left(u_{k+2}^{2}\right) o T^{-(k+2)}-2 h_{k+1} \cdot E\left(u_{k+1}^{2}\right) o T^{-(k+1)} \\
& \quad+h_{k} \cdot E\left(u_{k}^{2}\right) o T^{-k} d \mu \geq 0 \\
& \Leftrightarrow h_{k+2} \cdot E\left(u_{k+2}^{2}\right) o T^{-(k+2)}-2 h_{k+1} \cdot E\left(u_{k+1}^{2}\right) o T^{-(k+1)}+h_{k} \cdot E\left(u_{k}^{2}\right) o T^{-k} \geq 0 .
\end{aligned}
$$

Corollary 3. If $W$ be a weighted composition operator on $L^{2}(\mu)$ and $T^{-1}(\boldsymbol{A})=\boldsymbol{A}$. Then $W$ is k-quasi class $Q$ operator if and only if

$$
h_{k+2} \cdot u_{k+2}^{2} o T^{-2}-2 h_{k+1} \cdot u_{k+1}^{2} o T^{-1}+h_{k} \cdot u_{k}^{2} \geq 0
$$

Theorem 4.2. Let $W$ be a weighted composition operator on $L^{2}(\mu)$. Then $W$ is k-quasi * class $Q$ operator if and only if

$$
h_{k+2} \cdot E\left(u_{k+2}^{2}\right) o T^{-2}-2 h_{k} \cdot h o T^{-(k-1)} \cdot E\left(u_{k+1}^{2}\right)+h_{k} \cdot E\left(u_{k}^{2}\right) \geq 0 .
$$

Proof. Suppose W is k-quasi * class Q operator. Then for $f \in L^{2}(\mu)$.

$$
\left\langle\left(W^{* k} W^{* 2} W^{2} W^{k}-2 W^{* k} W W^{*} W^{k}+W^{* k} W^{k}\right) f, f\right\rangle \geq 0 .
$$


Let $f=\chi_{A}$ with $\mu(A)<\infty$. Then

$$
\begin{gathered}
\left\langle\left(W^{* k} W^{* 2} W^{2} W^{k}-2 W^{* k} W W^{*} W^{k}+W^{* k} W^{k}\right) \chi_{A}, \chi_{A}\right\rangle \geq 0 . \\
\Leftrightarrow\left\langle\left( h_{k+2} \cdot E\left(u_{k+2}^{2}\right) o T^{-(k+2)}-2 h_{k} \cdot E\left(u_{k+1}(h o T) E\left(u_{k+1}\right)\right) o T^{-k}\right.\right. \\
\left.\left.\quad+h_{k} \cdot E\left(u_{k}^{2}\right) o T^{-k}\right) \chi_{A}, \chi_{A}\right\rangle \geq 0 \\
\Leftrightarrow \quad h_{k+2} \cdot E\left(u_{k+2}^{2}\right) o T^{-(k+2)}-2 h_{k} \cdot E\left(u_{k+1}(h o T) E\left(u_{k+1}\right)\right) o T^{-k} \\
\left.\quad+h_{k} \cdot E\left(u_{k}^{2}\right) o T^{-k}\right) \chi_{A} d \mu \geq 0 \\
\Leftrightarrow \int_{A} h_{k+2} \cdot E\left(u_{k+2}^{2}\right) o T^{-(k+2)}-2 h_{k} \cdot E\left(u_{k+1}(h o T) E\left(u_{k+1}\right)\right) o T^{-k} \\
\quad+h_{k} \cdot E\left(u_{k}^{2}\right) o T^{-k} d \mu \geq 0 \\
\Leftrightarrow h_{k+2} \cdot E\left(u_{k+2}^{2}\right) o T^{-(2)}-2 h_{k} \cdot h o T^{-(k-1)} \cdot E\left(u_{k+1}^{2}\right)+h_{k} \cdot E\left(u_{k}^{2}\right) \geq 0 .
\end{gathered}
$$

Corollary 4. If $W$ be a weighted composition operator on $L^{2}(\mu)$ and $T^{-1}(\boldsymbol{A})=\boldsymbol{A}$. Then $W$ is $k$-quasi * class $Q$ operator if and only if

$$
h_{k+2} \cdot u_{k+2}^{2} o T^{-2}-2 h_{k} \cdot h o T^{-(k-1)} \cdot u_{k+1}^{2}+h_{k} \cdot u_{k}^{2} \geq 0 .
$$

Theorem 4.3. Let $T^{-1} \sum=\sum, W \in B\left(L^{2}(\mu)\right)$. Then $W$ is of quasi * class $Q$ if and only if

$$
f_{0}^{(3)} E\left(\pi_{3}^{2}\right) o T^{-3}-2\left(f_{0}^{(1)} E\left(\pi_{1}^{2}\right) o T^{-1}\right)^{2}+f_{0}^{(1)} E\left(\pi_{1}^{2}\right) o T^{-1} \geq 0
$$

Proof. Since $W$ is weighted composition operator with weight $\pi=\left(\frac{f_{0}}{f_{0} o T}\right)^{\frac{s}{2}}$ it follows that $W$ is of quasi * class Q operator if and only if $f_{0}^{(3)} E\left(\pi_{3}^{2}\right) o T^{-3}$ $2\left(f_{0}^{(1)} E\left(\pi_{1}^{2}\right) o T^{-1}\right)^{2}+f_{0}^{(1)} E\left(\pi_{1}^{2}\right) o T^{-1} \geq 0$.

The second Aluthge transformation of $\mathrm{T}$ described by B.P.Duggal is given by

$$
\tilde{T}=|\tilde{T}|^{\frac{1}{2}} V|\tilde{T}|^{\frac{1}{2}},
$$

where $\tilde{T}=V|\tilde{T}|$ is the polar decomposition of $\tilde{T}, \tilde{C}=|W|^{\frac{1}{2}}$, where $W=V|W|$ is the polar decomposition of the generalized Aluthge transformation $W: 0<$ $s<1$ is a weighted composition operator with weight

$$
\omega^{\prime}=J^{\frac{1}{4}} \pi\left(\frac{\chi_{\text {sup }}}{J^{\frac{1}{4}}} o T\right),
$$

where $J=f_{0} E\left(\pi^{2}\right) o T^{-1}$. 
Corollary 5. Let $T^{-1} \sum=\sum, W \in B\left(L^{2}(\mu)\right)$. Then $W$ is of quasi * class $Q$ if and only if $f_{0}^{(3)} E\left(\omega_{3}^{\prime 2}\right) o T^{-3}-2\left(f_{0}^{(1)} E\left(\omega_{1}^{\prime 2}\right) o T^{-1}\right)^{2}+f_{0}^{(1)} E\left(\omega_{1}^{\prime 2}\right) o T^{-1} \geq 0$

\section{5. k-quasi -*-class Q Composition Operator on Weighted Hardy Space}

The operator $C_{T}$ are not necessarily defined on all of $H^{2}(\beta)$. They are ever where defined in some special cases in the classical Hardy spaces $H^{2}$ (the case when $\beta_{n}=1$ for all $\mathrm{n}$ ).

Let $\omega$ be a point on the open disk. Define $k_{\omega}^{\beta}(z)=\sum_{n=0}^{\infty} \frac{z^{n} \bar{\omega}^{n}}{\beta_{n}^{2}}$. Then the function $k_{\omega}^{\beta}$ is a point evaluation for $H^{2}(\beta)$. Then $k_{\omega}^{\beta}$ is in $H^{2}(\beta)$ and $\left\|k_{\omega}^{\beta}\right\|^{2}=$ $\sum_{n=0}^{\infty} \frac{|\omega|^{2 n}}{\beta_{n}^{2}}$. Thus, $\left\|k_{\omega}\right\|$ is an increasing function of $|\omega|$. If $f(z)=\sum_{n=0}^{\infty} a_{n} z^{n}$ then $\left\langle f, k_{\omega}^{\beta}\right\rangle_{\beta}=f(\omega)$ for all $\mathrm{f}$ and $k_{\omega}^{\beta}$ is known as the point evaluation kernel at $\omega$. It can be easily shown that $C_{T}^{*} k_{\omega}^{\beta}=k_{T(\omega)}^{\beta}$ and $k_{0}^{\beta}=1$ (the function identically equal to 1 ).

Now we introduce the class of $\mathrm{k}$-quasi -* class $\mathrm{Q}$ operators, which is a common generalization of * class Q and quasi *-class Q operators, defined as follows: An operator $T \in B(H)$ is said to be $\mathrm{k}$-quasi-*-class $\mathrm{Q}$ operator if $T^{* k}\left(T^{* 2} T^{2}-2 T T^{*}+I\right) T^{k} \geq 0$, where $\mathrm{k}$ is a natural number.

Theorem 5.1. If $C_{T}$ is a $k$-quasi * class $Q$ operator in $H^{2}(\beta)$ then

$$
C_{T}^{*(k+2)} C_{T}^{k+2}-2 C_{T}^{* k} C_{T} C_{T}^{*} C_{T}^{k}+C_{T}^{* k} C_{T}^{k}=0 .
$$

Proof. An operator $C_{T}$ is k-quasi $*$ class $\mathrm{Q}$

$$
\begin{aligned}
& C_{T}^{*(k+2)} C_{T}^{k+2}-2 C_{T}^{* k} C_{T} C_{T}^{*} C_{T}^{k}+C_{T}^{* k} C_{T}^{k} \geq 0 \\
& \left\langle\left(C_{T}^{*(k+2)} C_{T}^{k+2}-2 C_{T}^{* k} C_{T} C_{T}^{*} C_{T}^{k}+C_{T}^{* k} C_{T}^{k}\right) f, f\right\rangle \geq 0 \\
& \left\langle\left(C_{T}^{*(k+2)} C_{T}^{k+2}\right) f, f\right\rangle-2\left\langle\left(C_{T}^{* k} C_{T} C_{T}^{*} C_{T}^{k}\right) f, f\right\rangle \\
& \quad+\left\langle\left(C_{T}^{* k} C_{T}^{k}\right) f, f\right\rangle \geq 0 \\
& \left\|C_{T}^{k+2} f\right\|^{2}-2\left\|C_{T}^{k} C_{T}^{*} f\right\|^{2}+\left\|C_{T}^{k} f\right\|^{2} \geq 0 \\
& \left\|C_{T}^{k+1}\left(C_{T} f\right)\right\|^{2}-2\left\|C_{T}^{k}\left(C_{T}^{*} f\right)\right\|^{2} \\
& +\left\|C_{T}^{k-1}\left(C_{T} f\right)\right\|^{2} \geq 0
\end{aligned}
$$




$$
\begin{aligned}
& \text { let } f=k_{0}^{\beta} \text { then } \\
& \qquad\left\|C_{T}^{k+1}\left(C_{T} k_{0}^{\beta}\right)\right\|^{2}-2\left\|C_{T}^{k}\left(C_{T}^{*} k_{0}^{\beta}\right)\right\|^{2}+\left\|C_{T}^{k-1}\left(C_{T} k_{0}^{\beta}\right)\right\|^{2} \geq 0 \\
& \left\|C_{T}^{k+1}\left(k_{0}^{\beta}\right)\right\|^{2}-2\left\|C_{T}^{k}\left(k_{T(0)}^{\beta}\right)\right\|^{2}+\left\|C_{T}^{k-1}\left(k_{0}^{\beta}\right)\right\|^{2} \geq 0
\end{aligned}
$$

Repeating the steps for $\mathrm{k}$ times and $T(0)=0$ we get

$$
\begin{aligned}
& \left\|k_{0}^{\beta}\right\|^{2}-2\left\|k_{0}^{\beta}\right\|^{2}+\left\|k_{0}^{\beta}\right\|^{2} \\
& =0 .
\end{aligned}
$$

Theorem 5.2. If $C_{T}^{*}$ is a $k$-quasi ${ }^{*}$ class $Q$ operator in $H^{2}(\beta)$ then

$$
C_{T}^{k+2} C_{T}^{*(k+2)}-2 C_{T}^{k} C_{T}^{*} C_{T} C_{T}^{* k}+C_{T}^{k} C_{T}^{* k}=0 .
$$

Proof. An operator $C_{T}^{*}$ is k-quasi $*$ class $\mathrm{Q}$

$$
\begin{aligned}
& C_{T}^{k+2} C_{T}^{*(k+2)}-2 C_{T}^{k} C_{T}^{*} C_{T} C_{T}^{* k}+C_{T}^{k} C_{T}^{* k} \geq 0 \\
& \left\langle\left(C_{T}^{k+2} C_{T}^{*(k+2)}-2 C_{T}^{k} C_{T}^{*} C_{T} C_{T}^{* k}+C_{T}^{k} C_{T}^{* k}\right) f, f\right\rangle \geq 0 \\
& \left\langle\left(C_{T}^{k+2} C_{T}^{*(k+2)}\right) f, f\right\rangle-2\left\langle\left(C_{T}^{k} C_{T}^{*} C_{T} C_{T}^{* k}\right) f, f\right\rangle+\left\langle\left(C_{T}^{k} C_{T}^{* k}\right) f, f\right\rangle \geq 0 \\
& \left\|C_{T}^{*(k+2)} f\right\|^{2}-2\left\|C_{T}^{* k} C_{T} f\right\|^{2}+\left\|C_{T}^{* k} f\right\|^{2} \geq 0 \\
& \left\|C_{T}^{*(k+1)}\left(C_{T}^{*} f\right)\right\|^{2}-2\left\|C_{T}^{* k}\left(C_{T} f\right)\right\|^{2}+\left\|C_{T}^{*(k-1)}\left(C_{T}^{*} f\right)\right\|^{2} \geq 0
\end{aligned}
$$

let $f=k_{0}^{\beta}$ then

$$
\begin{aligned}
& \left\|C_{T}^{*(k+1)}\left(C_{T}^{*} k_{0}^{\beta}\right)\right\|^{2}-2\left\|C_{T}^{* k}\left(C_{T} k_{0}^{\beta}\right)\right\|^{2}+\left\|C_{T}^{*(k-1)}\left(C_{T}^{*} k_{0}^{\beta}\right)\right\|^{2} \geq 0 \\
& \left\|C_{T}^{*(k+1)}\left(k_{T(0)}^{\beta}\right)\right\|^{2}-2\left\|C_{T}^{* k}\left(k_{0}^{\beta}\right)\right\|^{2}+\left\|C_{T}^{*(k-1)}\left(k_{T(0)}^{\beta}\right)\right\|^{2} \geq 0 .
\end{aligned}
$$

Repeating the steps for $\mathrm{k}$ times and $T(0)=0$ we get

$$
\begin{aligned}
& \left\|k_{0}^{\beta}\right\|^{2}-2\left\|k_{0}^{\beta}\right\|^{2}+\left\|k_{0}^{\beta}\right\|^{2} \\
& =0 .
\end{aligned}
$$

Example 5.1. Let $f=\sum_{n=0}^{\infty} f_{n} z^{n} \in H^{2}(\beta)$ and $\phi: D \rightarrow D$ be defined by $\phi(z)=\frac{e^{i \theta}}{2} z$, where $0 \leq \theta \leq 2 \pi$ is fixed. Then

$$
\left(C_{\phi}^{*} f\right)(\omega)=<C_{\phi}^{*} f, k_{\omega}>
$$




$$
\begin{aligned}
& =<f, k_{\omega o \phi}> \\
& =<f, k_{\varphi(\omega)}>\text { where } \varphi(\omega)=\frac{e^{-i \theta}}{2} \omega \\
& =f(\varphi(\omega)) \\
& =f\left(\frac{e^{-i \theta}}{2} \omega\right)
\end{aligned}
$$

Now

$$
\begin{aligned}
\left(C_{\phi}^{*} C_{\phi} f\right)(z) & =\left(C_{\phi} f\right)\left(\frac{e^{-i \theta}}{2} z\right) \\
& =f\left(\frac{e^{i \theta}}{2} \cdot \frac{e^{-i \theta}}{2} z\right) \\
& =f\left(\frac{1}{4} z\right)
\end{aligned}
$$

and

$$
\begin{gathered}
\left(C_{\phi} C_{\phi}^{*} f\right)(z)=\left(C_{\phi}^{*} f\right)\left(\frac{e^{i \theta}}{2} z\right) \\
=f\left(\frac{e^{-i \theta}}{2} \cdot \frac{e^{i \theta}}{2} z\right) \\
=f\left(\frac{1}{4} z\right) \\
\left(C_{\phi}^{*(k+2)} C_{\phi}^{k+2}-2 C_{\phi}^{* k} C_{\phi} C_{\phi}^{*} C_{\phi}^{k}+C_{\phi}^{* k} C_{\phi}^{k} f\right)(z) \geq 0 \\
\left(C_{\phi}^{*(k+2)} C_{\phi}^{k+2} f\right)(z)-2\left(C_{\phi}^{* k} C_{\phi} C_{\phi}^{*} C_{\phi}^{k} f\right)(z)+\left(C_{\phi}^{* k} C_{\phi}^{k} f\right)(z) \geq 0 \\
\left(C_{\phi}^{k+2} f\right)\left(\frac{e^{-i(k+2) \theta}}{2^{k+2}} z\right)-2\left(C_{\phi} C_{\phi}^{*} C_{\phi}^{k} f\right)\left(\frac{e^{-i(k) \theta}}{2^{k}} z\right)+\left(C_{\phi}^{k} f\right)\left(\frac{e^{-i(k) \theta}}{2^{k}} z\right) \geq 0 \\
f\left(\frac{e^{i(k+2) \theta}}{2^{k+2}} \frac{e^{-i(k+2) \theta}}{2^{k+2}} z\right)-2 f\left(\frac{e^{i(k) \theta}}{2^{k}} \frac{e^{-i \theta}}{2} \frac{e^{i \theta}}{2} \frac{e^{-i(k) \theta}}{2^{k}} z\right)+f\left(\frac{e^{i(k) \theta}}{2^{k}} \frac{e^{-i(k) \theta}}{2^{k}} z\right) \geq 0 \\
f\left(\frac{1}{2^{2 k+4}} z\right)-2 f\left(\frac{1}{2^{2 k+2}} z\right)+f\left(\frac{1}{2^{2 k}} z\right) \geq 0 .
\end{gathered}
$$

for every $f \in H^{2}(\beta)$ and $z \in D$. Hence $C_{\phi}$ is k quasi * class Q operator.

\section{References}

[1] Anuradha Gupta and Neha Bhatia, On (n,k)-Quasi Paranormal Weighted Composition Operator, International Journal of Pure and Applied Mathematics, 91, No. 1 (2014), 23-32. 
[2] J. Campbell and J. Jamison, On some classes of weighted composition operators, Glasgow Math. J., 32 (1990), 82-94.

[3] C. Cowen, Composition Operators on $H^{2}$, J. Operator theory, 9 (1983), 77-106.

[4] C. C. Cowen and T. L. Kriete, Subnormality and Composition Operators on $H^{2}$, Journal of functional Analysis, 81 (1988), 298-319.

[5] Carl C. Cowen, Linear Fractional Composition Operators on $H^{2}$, J. Integral Equality and Operator theory, 11 (1988), 151-160.

[6] B. P. Duggal, C. S. Kubrusly and N. Leven, Contractions of class Q and invariant subspaces, bull. J. Korean Math. Soc. , 42 (2005), 77-106.

[7] M. R. Embry, A generalization of the Halmos-Bram criterion for subnormality, Acta Sci. Math (Szeged), 35 (1973), 61-64.

[8] E. A. Nordgeen, P. Rosenthal and F. S. Wintrobe, Invertible Composition Operator on $H^{2}$, journal of functional Analysis, 73 (1987), 324-344.

[9] E. A. Nordgeen, Composition Operator in Hilbert space, In: Hilbert Space, Operators, Lecturer Notes in Math., J. Operator Theory, 693 (1977), 37-63.

[10] D. Kavitha, k-Quasi Class Q Composition Operators, International Journal of Pure and Applied Mathematics, 106, No. 7 (2016), 121-128.

[11] J. V. Ryff, Subordinate $H^{p}$ function, Duke Math. J., 33 (1966), 347-354.

[12] D. Senthilkumar, P. Maheswari Naik and R. Santhi, Weighted Composition of k-Quasi -Paranormal operators, International Journal of Matematical Archive, 3(2) (2012), 739746.

[13] S. Panayappan, D. Senthilkumar and R. Mohanraj, M-Quasi hyponormal Composition Operators on weighted Hardy spaces, Int. Journal of Math. Analysis, 2 (2008), 1163-1170.

[14] A. Lambert, Hyponormal Composition Operators, Bull. London Math. Soc. , 18 (1986), 125-134.

[15] T. Veluchamy and T. Thulasimani, Posinormal Composition Operators on Weighted Hardy space,International Mathematical forum, 5, No. 24 (2010), 1195-1205.

[16] J. Wolff Sur 1 iteration desfunctions, C. R. Acad. Sci. Pairs ser. A, 182 (1926), 42-43.

[17] J. T. Yuan and G. X. Ji, On (n,k)-quasi paranormal operators, Studia Math, 209 (2012), 289-301.

[18] Q. Zeng and H. Zhong, On (n,k)-quasi * paranormal operators, Studia Math (2012), 1-13. 
